OSCAR A. ROSAS-JAIMES ${ }^{1}$

(Corresponding author)

E-mail: oscar.rosasjaimes@yahoo.com

LUIS ALBERTO QUEZADA-TÉLLEZ ${ }^{2}$

E-mail: alquezada@ciencias.unam.mx

GUILLERMO FERNÁNDEZ-ANAYA ${ }^{2}$

E-mail: guillermo.fernandez@ibero.mx

${ }^{1}$ Facultad de Ciencias de la Electrónica,

Benemérita Universidad Autónoma de Puebla

Prolongacion 24 Sur S/N Ciudad Universitaria,

San Manuel, 72570 Puebla, México

2 Departmento de Física y Matemáticas,

Universidad Iberoamericana

Prol. Paseo de la Reforma 880, Álvaro Obregón,

Lomas de Sta. Fe, Cd. de México, México
Science in Traffic and Transport Original Scientific Paper

Submitted: Oct. 9, 2015

Accepted: Apr. 27, 2016

\title{
POLYNOMIAL APPROACH AND NON-LINEAR ANALYSIS FOR A TRAFFIC FUNDAMENTAL DIAGRAM
}

\begin{abstract}
Vehicular traffic can be modelled as a dynamic discrete form. As in many dynamic systems, the parameters modelling traffic can produce a number of different trajectories or orbits, and it is possible to depict different flow situations, including chaotic ones. In this paper, an approach to the wellknown density-flow fundamental diagram is suggested, using an analytical polynomial technique, in which coefficients are taken from significant values acting as the parameters of the traffic model. Depending on the values of these parameters, it can be seen how the traffic flow changes from stable endpoints to chaotic trajectories, with proper analysis in their stability features.
\end{abstract}

\section{KEY WORDS}

traffic fundamental diagram; nonlinear behaviour; polynomial approximation;

\section{INTRODUCTION}

Vehicular traffic studies have been carried out over the last few decades using average quantities, which can be obtained in many different and accurate ways in order to be analysed in different branches of traffic engineering $[1,2]$. These quantities are related in a well-known manner through what has been called a Fundamental Diagram [3, 4], which is often presented as a plot of the traffic flow-density relation. Such graphic representation, along with its mathematical expressions, is an idealization of the sets of points obtained from real measurements. Different mathematical expressions have been proposed to adjust them, recognized as good approximations which provide enough information for deterministic analysis.

In this paper, a polynomial approximation technique has been employed [5] to fit some significant points identified as those values that are common and important in every fundamental diagram, constituting a new and proper model to describe such a relation.

Additionally, this model has been modified in a discrete form, in such a way that it is possible to obtain different trajectories from an iterative scheme. This proposal has arisen because a similar approach has been attempted with the well-known logistic equation, taken as a valid traffic relation [6] but missing some specifications that a fundamental diagram does have, resulting in an interesting but incomplete analysis [12].

As we will show, distinct types of traffic situations can be analysed, spanning from those cases of a freeflow regime to congested-flow situations, where it is possible to find stable points or even cyclic events. There are some scenarios, however, where no pattern seems to exist in the traffic behaviour. Traffic values may oscillate, but in an unpredictable non-periodic way, cases in which traffic is said to develop chaotic behaviour [12].

The polynomial model presented here permits obtaining and illustrating all these behaviours by modifying a single parameter in it, the traffic average velocity $\mathrm{v}$, showing that vehicular traffic is a dynamic nonlinear system that also produces what is known as bifurcations and quasi-periodic trajectories [7].

\section{THE FUNDAMENTAL DIAGRAM}

A macroscopic approach to vehicular traffic focuses on aggregate variables, as mean velocities, flows, and vehicular densities. In this case, velocity $v$ is referred to as the speed of the wave front of vehicles, often considered to be the maximum speed reached by the average car moving in a free-flow, 
non-congested situation. From Figure 1 it is possible to observe that this quantity is very close in value to a tangent line from most of the values in the ascending part of the curve, but this feature changes as the flow approaches its maximum value $q_{\max }$. Congestion appears, and it can be more valuable to know the backward front of congestion velocity $w$, a quantity that measures the wave front that moves in the opposite direction to traffic flow, closely related to the descending part of the curve [3].

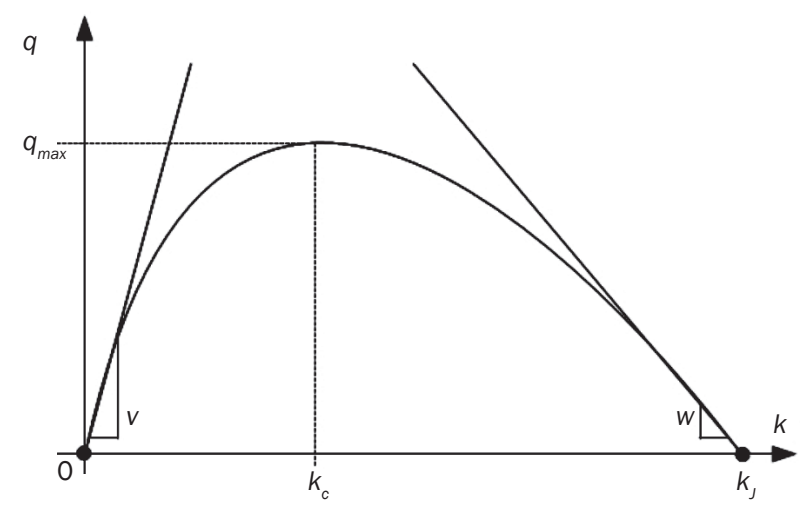

Figure 1 - Flow - Density (q vs. k) classic fundamental diagram

Flow $q$ is a quantity related to a set of vehicles moving with time. It can be a number of cars passing a point in a time interval or a set of cars traversing a section within a time interval $[1,3]$.

Density $k$ is defined as the number of vehicles occupying a section of a lane or stretch of a road. A direct measure of density can be obtained with aerial photographs, video images or in situ observations, i.e. limiting a length of a way and counting the vehicles present on it at the moment of time $[3,8]$.

These three quantities are simplistically related through $q=Q(k, v)$, and represented in Figure 1.

The form of this curve is rather descriptive and idealized. It depends on the particular cases of roads and their conditions. It is a complete and continuous function, but finding the whole range of values for each variable experimentally is not very likely. The data obtained in real life have multiple discontinuities, in which many parts of this curve are not present [9].

This curve illustrates several significant points. Note that null flow occurs in two different conditions:

1) When there are no cars on the road, density and flow are zero. The velocity will be that of the first driver to appear, generally a high value. This velocity is represented in the fundamental diagram as slope $v$ of a straight line passing through the origin, known as the free-flow velocity.

2) When density becomes so high that all vehicles are forced to stop, flow is zero again, as there is no movement. The density in this situation is known as jam density and it is referred to as $k_{j}$.

Between these two extremes there are many conditions of vehicular flow. As density increments from zero, flow does the same, as there are more cars on the road, but as this goes on velocity declines, because of the growing interactions between vehicles. This decrement in velocity is imperceptible when densities and flows are low or even medium, but increases noticeably as density $\mathrm{k}$ and flow $\mathrm{q}$ increase, removing the linear behaviour.

Velocity drop is remarkable before the flow reaches its maximum value. This condition is shown in Figure 1 in a point where the critical density $k_{C}$ and maximum flow $q_{\max }$ are found. Then velocity and flow decrease while density increases, starting the congested states in the fundamental diagram. Another important slope related to this portion of this curve is visible, corresponding directly to the backward front velocity of congestion $w$, and associated with a straight line plotted from the jam density point $k_{j}$ as depicted in Figure 1. As can be observed, any flow, except for the highest value, can occur in two different conditions, one with low density and another with high density.

\section{POLYNOMIAL APPROXIMATION}

The density-flow fundamental diagram is the best known plot in the vehicular traffic community, and one of the traffic system's most analysed features. Directly related to the LWR Hydrodynamic Model [10, 11], and confirmed by thousands of road measurements all over the world [1, 2, 3], it is the starting point for establishing traffic models [4].

Several authors have proposed mathematical expressions to achieve an approximation accurate enough to model the real phenomena and to perform theoretical analysis. Some of them are made from continuous functions $[6,13]$ and others are constructed in a piecewise manner [3]. All of them try to include the variables and parameters that are involved in the traffic phenomena in order to achieve a better fitness of the corresponding data that validate those models. This paper presents a polynomial approximation, with some remarkable advantages that will be introduced through its description and analysis in this paper.

In order to get an accurate approximation to a fundamental diagram, its main points and specifications as well have been taken into account (Figure 2). A three-degree polynomial has been proposed,

$$
q(k)=a_{0}+a_{1} k+a_{2} k^{2}+a_{3} k^{3}
$$

This function is chosen because of the non-symmetric form observed in real density-flow diagram, instead of a quadratic form that would necessarily suppose a symmetric parabola [6]. 
Equation 1 can be written in an equivalent factorized form,

$$
\begin{aligned}
q(k)= & a_{0}\left(k-k_{1}\right)\left(k-k_{2}\right)\left(k-k_{3}\right)+ \\
& +a_{1}\left(k-k_{0}\right)\left(k-k_{2}\right)\left(k-k_{3}\right)+ \\
& +a_{2}\left(k-k_{0}\right)\left(k-k_{1}\right)\left(k-k_{3}\right)+ \\
& +a_{3}\left(k-k_{0}\right)\left(k-k_{1}\right)\left(k-k_{2}\right)
\end{aligned}
$$

Coefficient $a_{0}$ can be obtained by making $k=k_{0}$

$$
\begin{aligned}
& q\left(k_{0}\right)=a_{0}\left(k_{0}-k_{1}\right)\left(k_{0}-k_{2}\right)\left(k_{0}-k_{3}\right) \\
& \Rightarrow a_{0}=\frac{q\left(k_{0}\right)}{\left(k_{0}-k_{1}\right)\left(k_{0}-k_{2}\right)\left(k_{0}-k_{3}\right)}
\end{aligned}
$$

Coefficients $a_{1}, a_{2}, a_{3}$ are obtained in a similar way, by making $k=k_{1}, k=k_{2}, k=k_{3}$, respectively and their substitution into Equation 2 can be written in the compact and general form,

$q(k)=\sum_{i=0}^{n} L_{i}(k) q\left(k_{i}\right)$

$L_{i}=\prod_{h=0}^{n} \frac{\left(k-k_{h}\right)}{k_{i}-k_{h}}$

with $i=0,1, \ldots n, h=0,1, \ldots n$ and $i \neq h$. The expressions 4 are known as Lagrange Polynomial [5], and for our specific case $n=3$. To perform a normalized analysis, let $k_{C}=1 / 4 k_{J}, v=4 w$ and $k_{J}=1$. These values and proportions are in agreement with those observed experimentally [3], i.e. with minimal variation, it has been observed that the backward wave velocity $w$ is approximately a quarter of its absolute value with respect to the magnitud of the free-flow velocity $v$.

Taking these facts into account, it is possible to propose values to calculate the coefficients of Equations 4, given in Table 1.

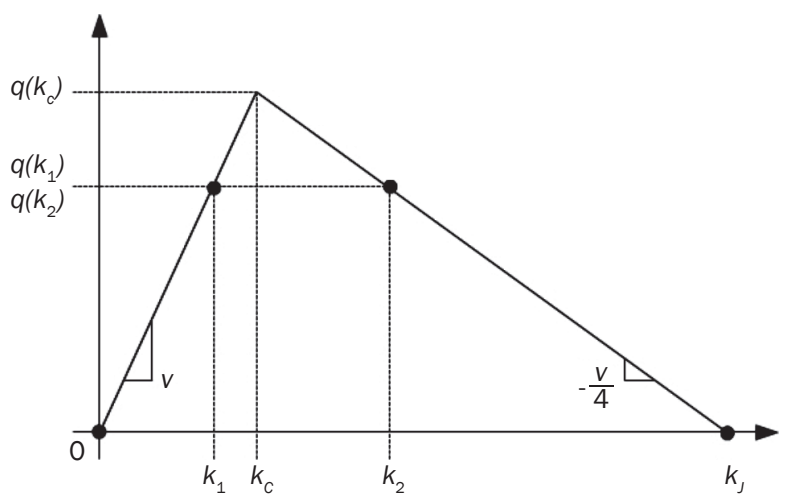

Figure 2 - Straight simplification of a normalized densityflow diagram $\left(k_{\jmath}=1\right)$, showing four main points and two lines with related slopes
Table 1 - Polynomial nodal points

\begin{tabular}{||c|c|c|}
\hline \hline$p$ & $k$ & $Q$ \\
\hline \hline 1 & $k_{0}=0$ & $q_{0}=0$ \\
\hline 2 & $k_{1}=\frac{3}{4} k_{c}=\frac{3}{16}$ & $q_{1}=q\left(k_{1}\right)=k_{1} v$ \\
\hline 3 & $k_{2}=k_{c}+4\left(k_{c}-k_{1}\right)$ & $q_{2}=q\left(k_{1}\right)=q\left(k_{2}\right)$ \\
\hline 4 & $k_{3}=k_{\lrcorner}=1$ & $q_{3}=0$ \\
\hline
\end{tabular}

The polynomial that touches the points $(0,0),\left(k_{1}\right.$, $\left.q\left(k_{2}\right)\right),\left(k_{2}, q\left(k_{2}\right)\right)$ and $\left(k_{\jmath}, 0\right)$ given in Table 1 is,

$$
\begin{aligned}
q(k)= & \frac{\left(k^{3}-\left(k_{\jmath}+k_{2}\right) k^{2}+k_{2} k_{\jmath} k\right)}{\left(k_{1}^{3}-\left(k_{\jmath}+k_{2}\right) k_{1}^{2}+k_{2} k_{\jmath} k_{1}\right)} q\left(k_{1}\right)+ \\
& +\frac{\left(k^{3}-\left(k_{\jmath}+k_{1}\right) k^{2}+k_{1} k_{\jmath} k\right)}{\left(k_{2}^{3}-\left(k_{\jmath}+k_{1}\right) k_{2}^{2}+k_{1} k_{\jmath} k_{2}\right)} q\left(k_{2}\right)
\end{aligned}
$$

Some advantages of this approach can be identified: points of minimum and maximum density and flow are taken into account in the design of the function representing the fundamental diagram, corresponding to measured and theoretical concepts.

On the other hand, it is noticeable that the following relations hold,

$q\left(k_{1}, v\right)=k_{1} v=\frac{3}{4} k_{c} v=\frac{3}{16} k_{\jmath} v$

From the specific cases of Equation 6, it is possible to note that given two of the variables the third can be calculated, but depending on these values the height, width, and general proportions of the curve change, and it is expected that the traffic behaviour represented by it changes as well. In this way, taking the values in Table 1, and those expressed by Equation 6, Equation 5 can be written as

$q(k, v)=\left(\frac{20}{13} k^{3}-\frac{159}{52} k^{2}+\frac{79}{52} k\right) v=P(k, v)$

Equation 7 is a concave curve as expected (Figure 3), the domain of which must be restricted to $0 \leq k \leq 1$ for the purposes of normalization of our model. The points where $k=0$ and $k=1$ are those two points where density is the lowest and the highest, as defined. This polynomial has a maximum at

$k_{c} \cong 0.331$

On the other hand, the vertical scale related to flow $q$ is not fixed in the same manner as the scale for density $k$, because it is affected by velocity $v$. This variable is bound by physical constraints related to the stream of vehicles unlike density related to road and its geometry.

Velocity $v$ is considered constant for a particular process, which means that such a quantity modifies the values of the coeficients in Equation 7 and therefore it is possible to work with a polynomial $Q(k)=P(k, v)$ of the density variable only, preserving proper units in the respective coefficients. 
$Q(k)=\left(\frac{20 v}{13} k^{3}-\frac{159 v}{52} k^{2}+\frac{79 v}{52} k\right)$

Equation 9 preserves the shape of the fundamental diagram as depicted in Figure 3 , but it depends only on a single variable. By the normalization made to this variable, where $k \in[0,1]$, it is possible to see that $Q(k)$ $\in[0,1]$ also. Then it is also possible to perform an iterative process of the form $k_{i+1}=Q\left(k_{i}\right)$, where index $i$ is now used to indicate this iterative process, preserving all the properties of the obtained polynomial, but treating it as a discrete dynamic system, and therefore, resulting in the following expression

$$
k_{i+1}=\left(\frac{20 v}{13} k_{i}^{3}-\frac{159 v}{52} k_{i}^{2}+\frac{79 v}{52} k_{i}\right)=Q\left(k_{i}\right)
$$

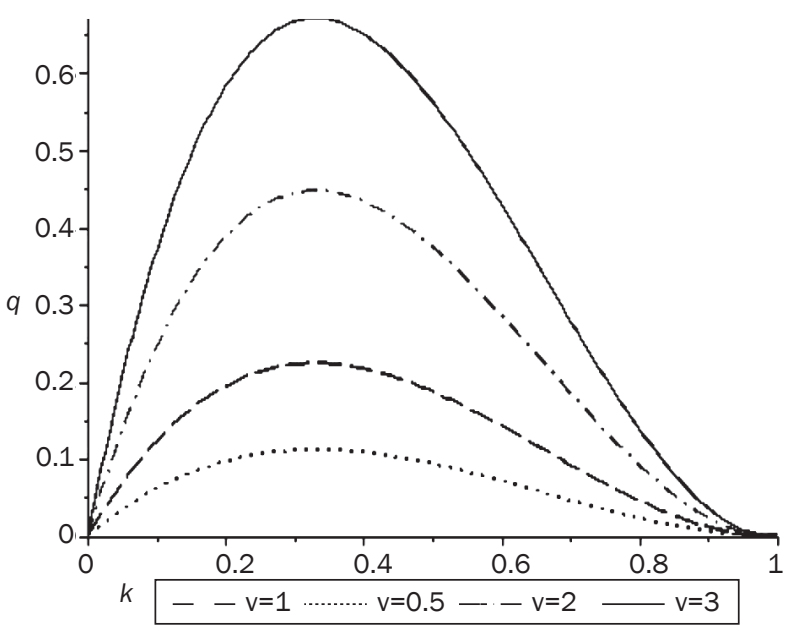

Figure 3 - Polynomial shapes, from Equation (7), as velocity $v$ is varied

The actual values of $k_{i}$ on Equation 10 condition the next state $k_{i+1}$, i.e. by starting from an initial condition $k_{0}$, Equation 10 will generate a new value $k_{1}$, continuing in a sequence $\left\{k_{n}\right\}=\left\{k_{0}, k_{1} \ldots k_{i+1}\right\}$ as other works have done with logistic-type functions [7, 8, 12].

As claimed in the Introduction of this paper, one of the main purposes of this work is to adjust the shape of the curve used to perform the same iterative processes that appear in such publications, where the iterations are performed over one-dimension functions with expressions such as $X_{i+1}=F\left(X_{i}\right)$. Even though Equation 10 comes evidently from a two-dimension function, due to the normalization that preserves the properties of the fundamental diagram restricted to a unit square [7, 11], the processes are valid.

\section{STABILITY ANALYSIS}

\subsection{Fixed points}

From Equation 10 it is possible to calculate some interesting properties of this discrete model. To do it, some useful definitions and propositions are needed first.

Definition 1. If $Q$ is a function in such a way that $Q\left(k^{*}\right)=k^{*}$, then $k^{*}$ is a fixed point of $Q[7,12]$.

In other words, Equation 10 has a fixed point $k^{*}=k_{i}$ if the point $\left(k_{i}, k_{i}\right)$ exists on the plot of the iteration-generated set, i.e. the fixed point also exists in a $45^{\circ}$ line over the same plot of the discrete system.

Taking into account Definition 1, from Equation 10 it is possible to obtain three possible fixed points $k^{*}$ for density $k$, being $k^{*}=0$ one of them, trivially obtained, while the other two fixed points can result from Equation 11, which results in terms of the velocity $v$ only.

$k^{*}=\frac{159}{160} \pm \frac{1}{160} \sqrt{\frac{1}{v}(v+16640)}$

Since the proposed polynomial is a third degree expression, only these three fixed points are expected each time a new iterative process is calculated, by changing velocity $v$ in each one of such processes. Figure 4 depicts the behaviour for such fixed points. However, because the effective set of density values is $0 \leq k \leq 1$, one of the fixed points $\left(k_{1}{ }^{*}\right.$ in Figure 4$)$ is not useful for our analyses, because $k_{1}{ }^{*}>1$. On the other hand, there are two fixed points whose range of values are included in such an interval, $k_{2}{ }^{*}$ (as indicated in Figure 4), which is effective from $v \mid k_{2}^{*}=0 \cong 0.658$ and $k_{3}{ }^{*}$ (as shown in Figure 4 ) which is always 0 . These fixed points signal those values where density $k$ is affected by velocity $v$, producing different behaviours on the evolution of this variable.

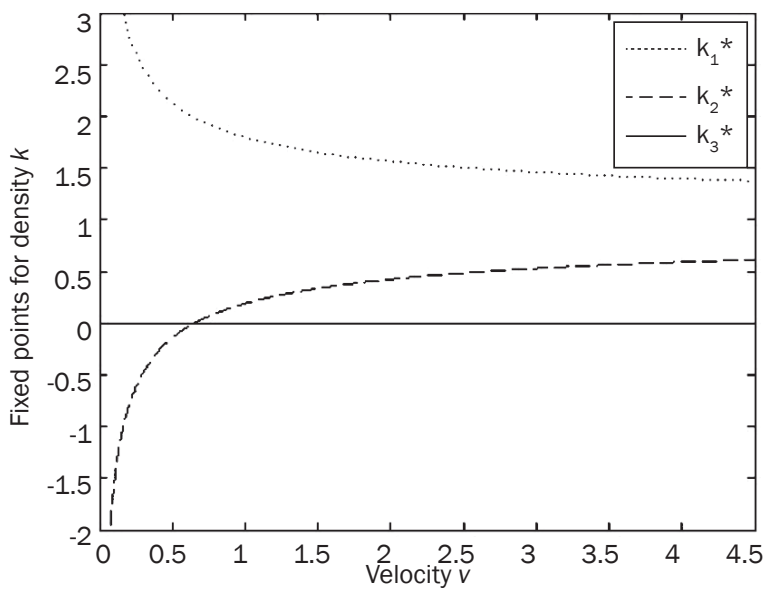

Figure 4 - Fixed points for the proposed model, as velocity $v$ is varied

\subsection{Behavioural intervals}

Definition 2. A point $\mathrm{k}$ is known as a periodic point [7, 12] of function $Q$ with period $m$ if $Q^{m}(k)=k$, i. e. $k$ is a periodic point of $Q$ with period $m$ if $k$ is a fixed point of $Q^{m}$.

Point $k$ has prime period $m_{0}$ if $Q^{m 0}(k)=k$ and $Q^{n}(k) \neq k$ whenever $0<n<m_{0}$, i. e. $k$ has prime period $m_{0}$ if $k$ returns back to a previous point for the first time after $m_{0}$ iterations of Q. 
The set of all iterations of $k$ is called the orbit of $k$ and if $k$ is a periodic point then the set of its iterations is called a periodic orbit or a cycle.

Definition 3. Let $Q$ be a function and $p$ a periodic point of $Q$ with prime period $m$. Then $k$ is forward asymptotic to $p$ if there is a sequence $k, Q^{m}(\mathrm{k}), Q^{2 m}(\mathrm{k}), Q^{3 m}(\mathrm{k})$, that converges to $p$, i.e. $\log _{n \rightarrow \infty} Q^{n m}(k)=p$. The stable set of $p$, denoted by $\Omega s(p)$, includes all of the points which are forward asymptotic to $p[7,12]$.

Theorem 1. Let $Q$ be a continuous and derivable function and let $p$ be a fixed point of $Q$. Then, the absolute value of the derivative of $Q$ in $p,\left|Q^{\prime}(p)\right|<1$ implies that there is a neighbourhood around $p$ which is contained in $\Omega s(p)$ and $\left|Q^{\prime}(p)\right|>1$ implies that there is a neighbourhood around $p$ whose points make the values of $Q$ to leave under iteration. The proof of this Theorem can be found in reference [12].

Definition 4. Let $\mathrm{p}$ be a periodic point of $Q$ with prime period $m$. If $\left|\left(Q^{m}\right)^{\prime}(p)\right|<1$, then $p$ is an attracting periodic point of $Q$. If $\left|\left(Q^{m}\right)^{\prime}(p)\right|>1$, then $p$ is a repelling periodic point of $Q[7,12]$.

Definition 5. [15] The zero solution of Equation 10 is said to be exponentially stable if there is $M \geq 0$ and $\beta \in[0,1)$ such that

$$
\begin{aligned}
& \forall \alpha, \alpha_{0} \in \mathbb{Z}_{+}, \alpha \geq \alpha_{0} ; \\
& \forall k^{*} \in \mathbb{R}^{n}:\left\|Q\left(\alpha, \alpha_{0}, k^{*}\right)\right\| \leq M \beta^{\alpha-\alpha_{0}}\left\|k^{*}\right\|
\end{aligned}
$$

Theorem 2. Every fixed point $k^{*}$ in polynomial 10 is exponentially stable.

Proof: consider polynomial $Q(k)$ in Equation 10 and consider its derivative form

$Q^{\prime}=\left(\frac{60}{13} k_{i}^{2}-\frac{159}{26} k_{i}+\frac{79}{52}\right) v$

If $k^{*}$ is a fixed point and if $\left|Q\left(k^{*}\right)\right|<1$ then

$\lim _{k \rightarrow k^{*}}\left|\frac{Q(k)-Q\left(k^{*}\right)}{k-k^{*}}\right|<1$

For a fixed point $Q\left(k^{*}\right)=k^{*}$

$\lim _{k \rightarrow k^{*}}\left|\frac{Q(k)-k^{*}}{k-k^{*}}\right|<1$

Because polynomial $Q(k, v)$ is continuous and its derivative in $k^{*}$ exists, then

$\lim _{k \rightarrow k^{*}}\left|\frac{Q(k)-k^{*}}{k-k^{*}}\right|<M \beta^{\alpha-\alpha_{0}} k^{*}<1$

There is a $\delta>0$ such that $k \in\left(k^{*}-\delta, k^{*}+\delta\right) \cap[0,1]$ and therefore

$\left|\frac{Q(k)-k^{*}}{k-k^{*}}\right|<M \beta^{\alpha-\alpha 0} k^{*}<C<1$

Thus

$\left|Q(k)-k^{*}\right|<C\left|k-k^{*}\right|$

As the distance from $k$ to $k^{*}$ is less than $\delta$, i.e. $\left|Q(k)-k^{*}\right|<\delta$, then

$Q\left(\left(k^{*}-\delta, k^{*}+\delta\right) \cap[0,1]\right) \subset\left(k^{*}-\delta, k^{*}+\delta\right) \cap[0,1]$
This can be extended to any iteration number $n \in \mathbb{N}$

$\left|Q_{n}(k)-k^{*}\right|<C^{n}\left|k-k^{*}\right|$

Therefore, $\quad \forall k \in\left(k^{*}-\delta, k^{*}+\delta\right) \cap[0,1], \quad$ iterative polynomial $Q_{n}(k)$ is exponentially stable and then $Q_{n}(k) \rightarrow k^{*}$. QED

\section{DYNAMIC BEHAVIOUR}

From Theorems 1 and 2 it is possible to know the way in which the convergence to a fixed point is performed around the maximum of the density. In fact, if the fixed point is before the maximum of density, then the derivative of $Q$ is positive, $0<M<1$, and the iterations behave in a monotonic fashion. If the fixed point is located after the maximum of density, then the derivative of $Q$ is negative, $-1<M<0$, and the iterations behave in a cyclic way.

This behaviour is not independent from the value of velocity $v$. In fact, from the calculations plotted in Figure 4 it is possible to see that for $v \rightarrow 0$, fixed point $k^{*}=0\left(k_{3}{ }^{*}\right.$ in Figure 4$)$ is an attracting periodic point in a forward asymptotic behaviour as stated by Definition 3 and 4 , as there is no other fixed point in the interval $k \in[0,1]$. When starting with an initial density of $k=0.2$ and a velocity $v=0.50$, the behaviour of the iterated trajectory is towards zero monotonically (Figure 5), as mentioned. This situation is closely related to a traffic road observed with initial values of low density of vehicles evolving toward an empty condition, a situation mostly observed during those hours between the night and the next morning.

Increasing velocity $v$, reaching $v \mid k_{2}^{*}=0 \cong 0.658$, a second fixed point $\left(k_{2}{ }^{*}\right.$ in Figure 4$)$ appears and Theorem 2 can be validated for $0 \leq k_{2}^{*} \leq 0.5$ and for all $k_{3}{ }^{*}$. In this case $k_{3}{ }^{*}$ is a repelling periodic point and $k_{2}{ }^{*}$ is an attracting one (Definition 4), in a forward asymptotic manner also (Definition 3). When starting again with an initial density of $k=0.2$ but with velocity $v=0.75$, the behaviour of the iterated trajectory moves away the origin and now approaches the fixed point $k=0.063 \mathrm{ap}$ proximately (Figure 6), also in a monotonic manner, in agreement with the definitions and analyses annotated in the last section. This is a situation that relates to those real circumstances where evolution of the traffic goes to stable values of low densities.

Cases depicted by Figures 5 and 6 have in common velocity $v$ that affects the shape of the polynomial in such a way that the $45^{\circ}$ line does not surpass the maximum value for this function $k \cong 0.331$ when crossing it. With this value of $\mathrm{k}$ and from Equation 11 , it is possible to obtain $v=1.481$ as the limit value where the behaviour of the trajectories changes to oscillations, but it still behaves as exponentially stable in agreement with Theorem 2. 


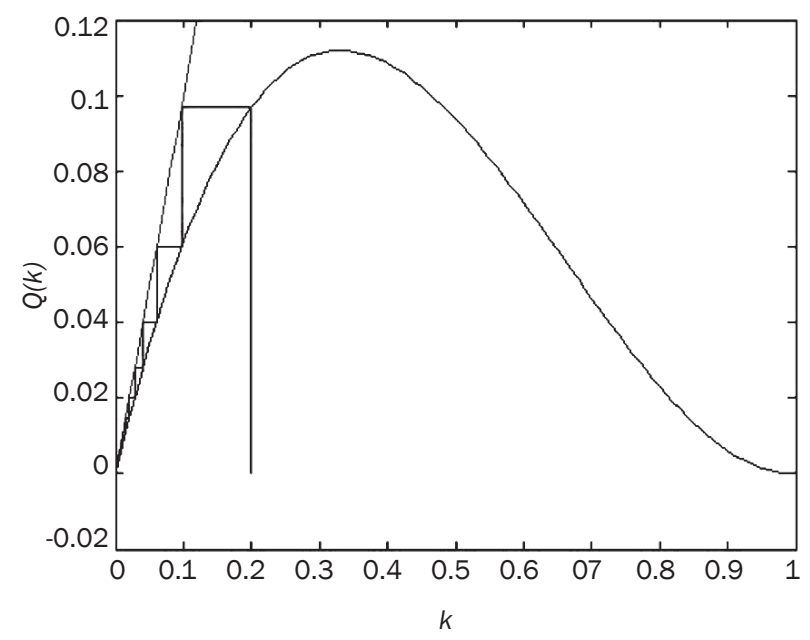

Figure 5 - Trajectory for $v=0.50$ (initial density $k=0.2$, final value $k=0$ )

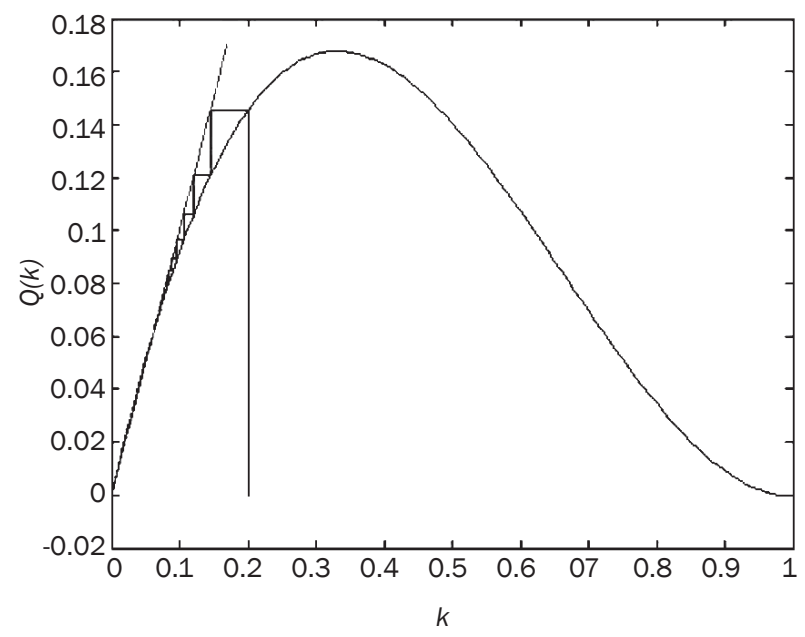

Figure 6 -Trajectory for $v=0.75$ (initial density $k=0.2$ final value $k=0.628$ )

This can be seen in Figure 7 , for velocity $v=2.40$, with initial density $k=0.3$. In this case, the attracting fixed point is $k=0.473$ approximately. As in the last two cases, traffic evolves to a stable condition, but in this specific situation traffic is congested, because density values are high in comparison, and the flow starts to tend towards low values. On the other hand, in these three cases depicted here, stability in traffic is shown as a set of points that converge towards a single end point. Even though they can be started from different initial conditions, it can be verified that the form of the trajectories will be practically the same after the transients vanish.

It is useful to plot another figure in which final values are drawn against the velocity $v$ value from which they were generated. Figure 13 shows the positions for those final values. In this way, it is possible to see that from $v=0$ to $v \cong 0.658$ all the trajectories fall towards zero, as was already mentioned for this interval. After this value of $\boldsymbol{v}$, the possible trajectories also converge to an attracting fixed value, which increases as velocity $v$ also increases.

When velocity $v$ increases to $v=\frac{8}{3} \cong 2.667$, the corresponding fixed point is $k_{2}^{*}=\frac{1}{2}$, and it is no longer possible to verify the result of Theorem 2. Even though it is possible to watch a stable behaviour, this is distinct in nature, marking a different kind of iteration process. Figure 8 shows a trajectory that does not converge to a single point, but a limit cycle. Here, $v=2.90$ and it is possible to see in Figure 13 that this value corresponds to a region where two final values of the polynomial 10 can be obtained, which is in correspondence with the periodical trajectory found. In other words, this trajectory, or orbit, will exhibit $k=0.346$ and $k=0.648$ for any pair of future iterations after stabilization. Then, as stated by Definition 2 , it has prime period $m_{0}=2$. Notice that the corresponding fixed value is in agreement with the calculations that are depicted in Figure 4 from Equation 11.

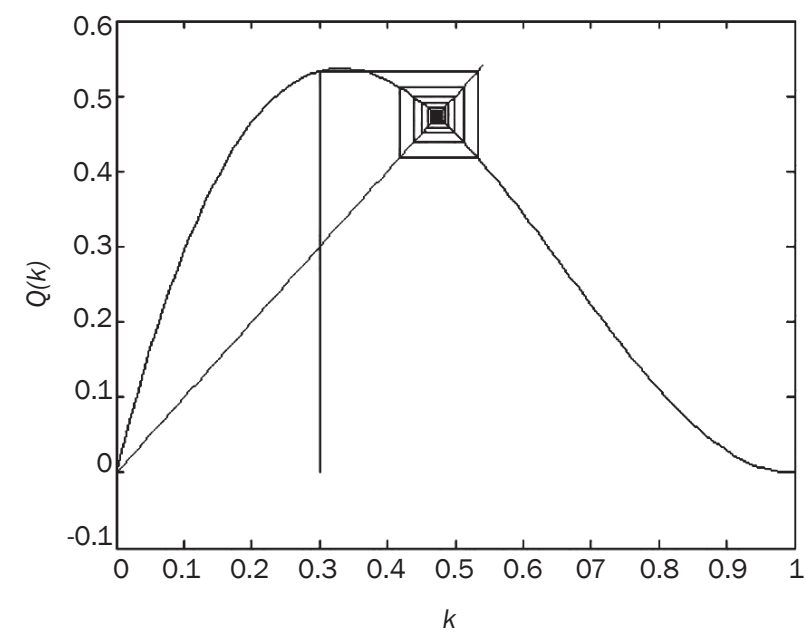

Figure 7 - Trajectory for $v=2.40$ (initial density $k=0.3$, final value $k=0.473$ approximately)

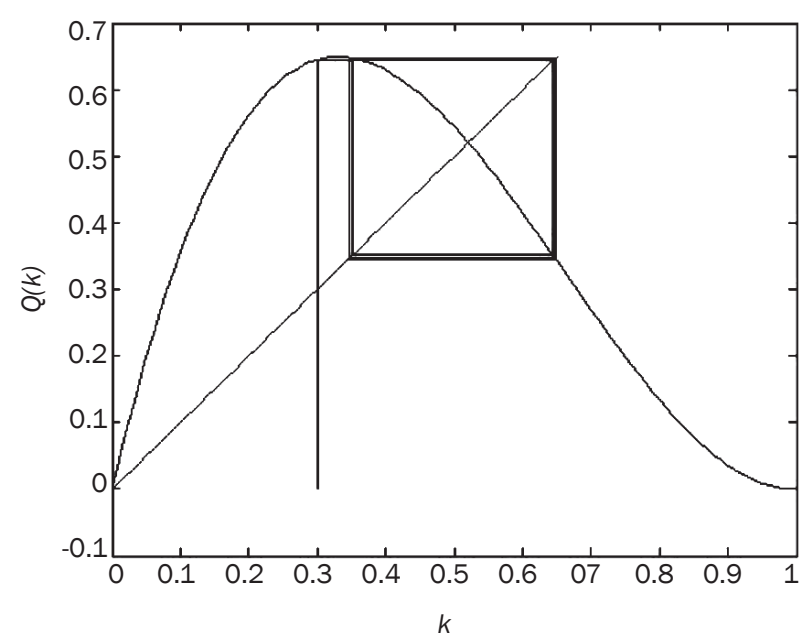

Figure 8 - Trajectory for $v=2.90$ (initial density $k=0.3$, final values $k=0.346$ and $k=0.648$ approximately) 
It is important to note that such behaviour is also observed in real traffic [3].

This cyclic phenomenon increases its complexity as $v$ is varied as Figure 9 shows. From such a mapping, if velocity is increased to approximately $v=3.3$ periodicity changes. Figure 9 depicts a limit cycle with $m_{0}=4$ and values: $k=0.239, k=0.697, k=0.314$ and $k=0.743$. As seen in Figure 13 this value of $v$ corresponds to a region where there are four branches which split from the anterior two-branch region. If this process is further repeated, now for $v=3.45$ (Figure 10), $m_{0}=8$, and the bifurcation map is in a region of eight branches (Figure 13), with final cyclic orbits with values of $k=0.343, k=0.771, k=0.203, k=0.674, k=0.366$, $k=0.766, k=0.212$ and $k=0.687$ approximately.

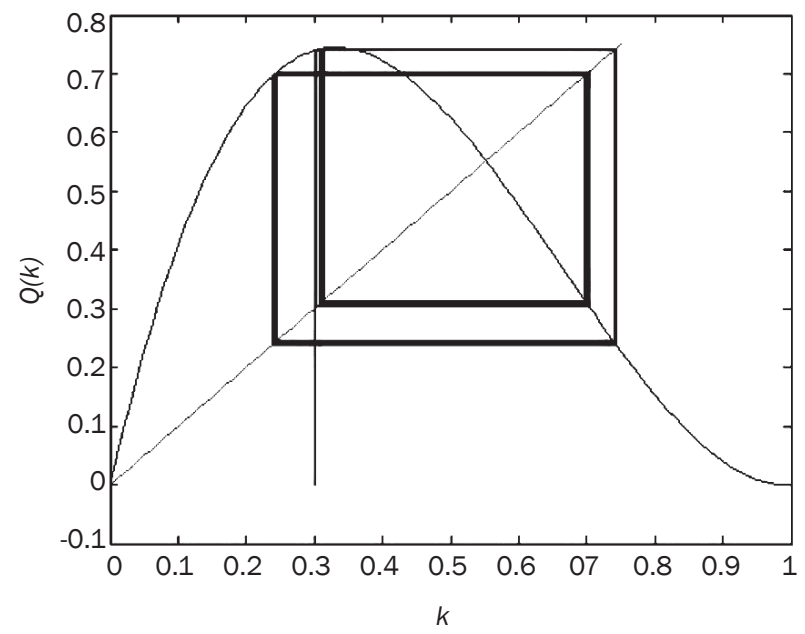

Figure 9 - Trajectory for $v=3.33$ (initial density $k=0.3$, final values $k=0.239, k=0.697, k=0.314$ and $k=0.743$ approximately)

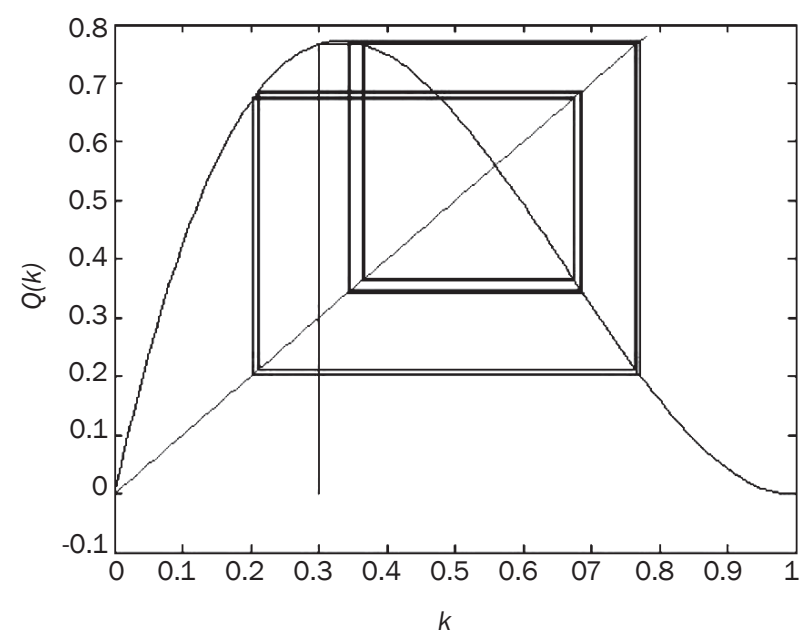

Figure 10 - Trajectory for $v=3.45$ (initial density $k=0.3$, final values $k=0.343, k=0.771, k=0.203, k=0.674, k=$ $0.366, k=0.766, k=0.212, k=0.687$ )
If this process is further repeated, now for $v=3.45$ (Figure 10), $m_{0}=8$, and the bifurcation map is in the region of eight branches (Figure 13), with final cyclic orbits with values of $k=q=0.343, k=q=0.771, k=0.203$, $k=0.674, k=0.366, k=0.766, k=0.212$ and $k=0.687$ approximately. In real situations, these last three cases would correspond to situations that change from almost optimum values of flow to severe congestion in an oscillating manner as noted in real scenarios [3].

As it is already known for similar iterative schemes $[6,7,12]$, this cyclic behaviour doubles its period in a manner described by the mapping in Figure 13. Velocity $v$ is a parameter that triggers such behaviour as established now. It is possible to find more of these regions that double the periodicity as the velocity parameter increases.

However, as can be seen in the same Figure 13, the size of intervals decreases progressively and then an $n$-periodicity will merge easily with the next $2 n$-periodicity and rapidly with the next doubling periodicity region. This behaviour is technically deterministic but practically unpredictable, and it is said that a chaotic system has been found.

For $v=3.9$ the system is in a chaotic region, with undetermined periodicity, which means that even though this system is deterministic, as it follows a well-defined mathematical model, deviations in trajectories that start very close will magnify as iterations pass. For example, Figure 11 shows the polynomial with $v=3.9$, beginning at $k=0.7$, and after a number of 120 iterations the final value reached is $k=0.408$. For a slight deviation in the initial condition, namely $k=0.701$ (Figure 12), leaving all other values equal, the final value obtained is $k=0.777$.

This quantity will be different if a different number of iterations are performed, with no periodicity identified. This happens inside the boundaries that the same systems have, that is to say, none of the trajectories will tend to infinity. Unlike other situations shown, where no deviation in final values is expected for slight differences in the initial conditions, two chaotic systems are characterized by growing differences in their final values as iterations pass. The trajectories themselves are seen to behave differently if a detailed comparison is made of both figures. 


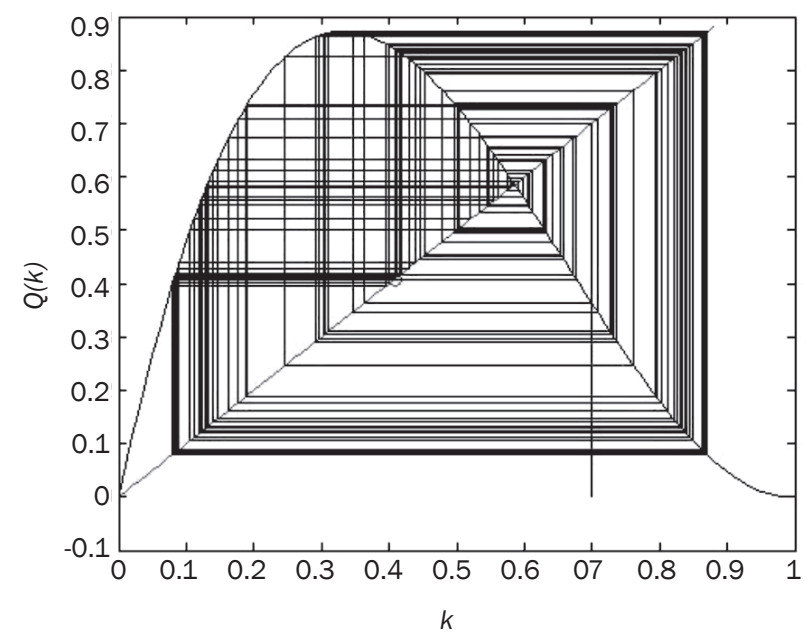

Figure 11 - Trajectory for $v=3.90$ (initial density $k=0.7$, final values $k=0.408$ approximately)

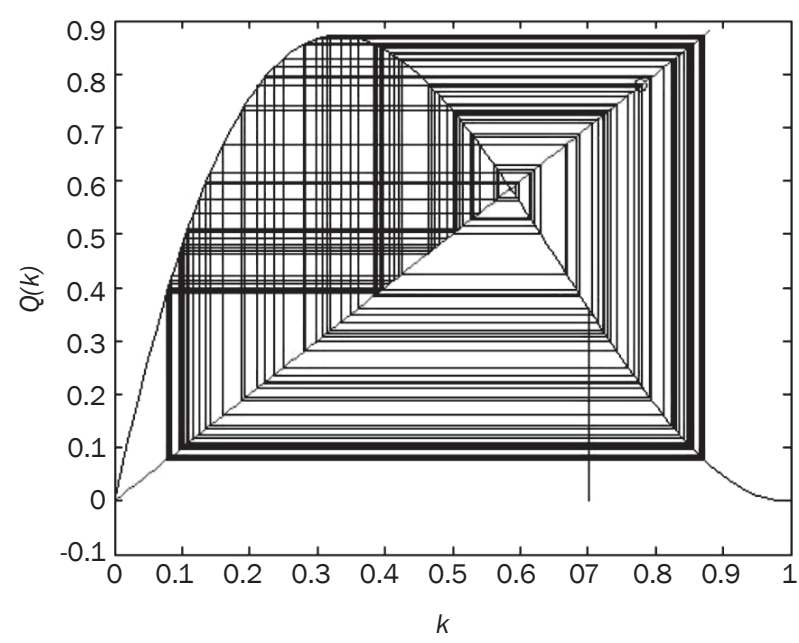

Figure 12 - Trajectory for $v=3.90$ (initial density $k=0.701$, final values $k=0.777$ approximately)

Figure 13 shows its own features by itself. Between approximately values $v=3.9$ and $v=4.1$, it seems that periodicity is recovered for some periodic values, but suddenly aperiodicity appears again after some short regions of bifurcations that are not well defined.

As has been shown, all these behaviours have been dependent of the variation of velocity $v$. Each time this parameter increases, the shape of the polynomial 9 changes. This condition has a limit and it is possible to calculate the highest value for velocity as $v=4.47$, reached when $Q\left(k_{i}\right)=1$ at the maximum of the normalized fundamental diagram when density $k \cong 0.331$.

\section{CONCLUSIONS}

A three-degree polynomial approach to obtain a model of a fundamental diagram is presented, and its accuracy depends on the important points of this type of traffic behaviour representation.

It has been shown that for some sets of values of velocity $v$, the behaviour is stable with states that approach a single end value, or one with periodic values. The periodicity can be only 2 if the velocity parameter $v$ lies in certain interval or 4 if this parameter increases. It can be found that this periodicity doubles to 8,16 or more for higher values of $v$. The periodicity becomes so high that it is impossible to predict a final value for two sets of iterations performed with slightly different initial conditions, as their trajectories diverge. This traffic model is said to have become chaotic.

This diffuse transition between bifurcations, which correspond to oscillatory convergence as has been seen, is a different feature with respect to the use of two-degree polynomial that is the logistic equation used in [8], for example. The suggested polynomial approach has the advantage of showing convergent oscillatory phenomena, expected in real situations, besides those described by the parabolic polynomial already mentioned.

It has the additional advantage of being more closely related to the fundamental diagrams that are well known in traffic fields, as its design is based on nodal points, related to some important concepts in traffic theory. These points and the approximation given by the Lagrange polynomial of a higher degree avoid an unrealistic symmetry and approach a more familiar fundamental diagram.

There are other functions that approach data depicting the fundamental diagram, proposed by other researchers (Greenshields, Greenberg, Underwood, etc.). The common feature of all of them is that they take nodal points as the basis to design and to suggest such models, in a similar manner in which our polynomial has been constructed. It is not strange then that all the definitions, analyses and simulations performed here in this paper can be made on those other models. This is part of our future work on which we will be focusing.

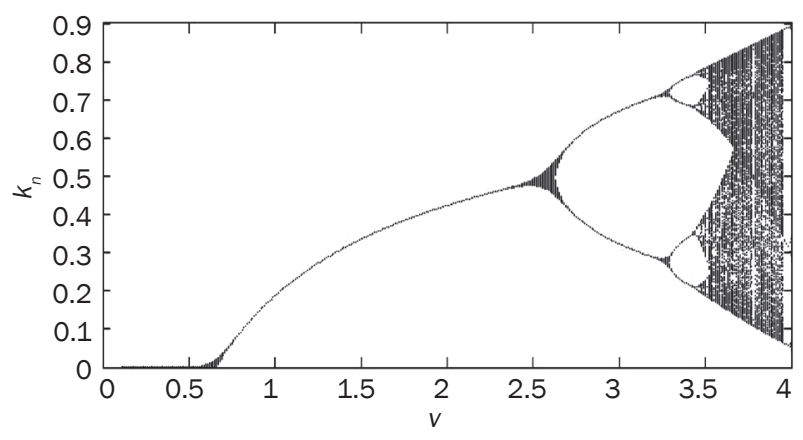

Figure 13 - Density-Flow Mapping as velocity $v$ changes. 
OSCAR A. ROSAS-JAIMES ${ }^{1}$

(Corresponding author)

E-mail: oscar.rosasj@correo.buap.mx

LUIS ALBERTO QUEZADA-TÉLLEZ ${ }^{2}$

E-mail: alquezada@ciencias.unam.mx

GUILLERMO FERNÁNDEZ-ANAYA ${ }^{2}$

E-mail: guillermo.fernandez@ibero.mx

${ }^{1}$ Facultad de Ciencias de la Electrónica,

Benemérita Universidad Autónoma de Puebla

Prolongacion 24 Sur S/N Ciudad Universitaria,

San Manuel, 72570 Puebla, México

2 Departmento de Física y Matemáticas,

Universidad Iberoamericana

Prol. Paseo de la Reforma 880, Álvaro Obregón,

Lomas de Sta. Fe, Cd. de México, México

\section{ENFOQUE POLINOMIAL Y ANÁLISIS NO LINEAL PARA UN DIAGRAMA FUNDAMENTAL DE TRÁFICO VEHICULAR}

\section{RESUMEN}

El tráfico vehicular puede modelarse como una forma dinámica discreta. Como en muchos sistemas dinámicos, los parámetros que modelan tráfico pueden producir un número de diferentes trayectorias $u$ órbitas $y$ es posible representar diferentes situaciones de flujo, incluyendo las que presentan caos. En este documento, un enfoque sobre el bien conocido diagrama fundamental de densidad-flujo es propuesto, usando una técnica polinomial analítica, en la cual los coeficientes se toman a partir de valores significativos que actúan como los parámetros del modelo de tráfico. Dependiendo de los valores de estos parámetros, se puede ver como el flujo de tráfico cambia de puntos finales estables a trayectorias caóticas, con el análisis apropiado en sus características de estabilidad.

\section{PALABRAS CLAVE}

diagrama fundamental de tráfico vehicular; comportamiento no lineal; aproximación polinomial;

\section{REFERENCES}

[1] Institute of Transportation Engineers (ITE) Traffic Engineering Handbook 6th ed. Washington DC, 2009.

[2] Payne H., Models of freeway traffic and control, in Mathematic Models of Public Systems. Smulation Council, 1971; 28(1):51-61.

[3] Daganzo C. F., Fundamentals of Transportation and Traffic Operations, Pergamon, Elsevier.

[4] Marušić S., Fluid Models in the Traffic Flow Theory, Promet - Traffic \& Transportation, 2000; 12(1): 7-14.

[5] Chapra S. and Canale R., Numerical Methods for Engineers, 6th Ed. McGraw-Hill, 2009.

[6] Lo S.-C. and Cho H.-J., Chaos and control of discrete dynamic model, Journal of the Franklin Institute, 2005; 342: 839-851.

[7] Devaney R. L., An introduction to chaotic dynamical systems, 1987.

[8] Thamizh V. A. and Dhivya G., Measuring heterogeneous traffic density, International Journal of Engineering and Applied Sciences, 2010; 6(3): 144-148.

[9] Kim T. and Zhang H. M., An empirical study on gap time and its relation to the fundamental diagram of traffic flow, in 7th International IEEE Conference on Intelligent Transportation Systems, Washington, D.C., 2004: 94-99.

[10] Lighthill M. J. and Whitham G. B., On kinematic waves. I. Flood movement in long rivers, Proc. Royal Soc. A., 1955; 229: 281-316.

[11] Richards P. I., Shock waves on the highway, Operation research, 1956; 4: 42-51.

[12] Holmgren, R. A., A first Course in Discrete Dynamical Systems, Springer, N. Y., 1994.

[13] Greenberg, H., An analysis of traffic flow. Operations Research 1959; 7: 79-85.

[14] Greenshields, B.D. "A study of traffic capacity". Highway Research Board, 1935; 14: 448-477.

[15] Ngoc P.H.A., Hieu L.T., On stability of discrete-time systems under nonlinear time-varying perturbations, Advance in Difference Equations 2012, 2012:120. 